\title{
Nouvelles formes de gouvernance dans le domaine de l'eau. Apports et limites de la coopération décentralisée dans les pays en développement
}

New Forms of Governance in the Water Sector. Contributions and Limitations of Decentralized Cooperation in Developing Countries

Raoudha Makkaoui et Jean Luc Dubois

\section{OpenEdition}

\section{Journals}

Édition électronique

URL : http://journals.openedition.org/developpementdurable/8413

DOI : 10.4000/developpementdurable.8413

ISSN : 1772-9971

Éditeur

Association DD\&T

Référence électronique

Raoudha Makkaoui et Jean Luc Dubois, « Nouvelles formes de gouvernance dans le domaine de l'eau. Apports et limites de la coopération décentralisée dans les pays en développement », Développement durable et territoires [En ligne], Vol. 1, n 1 | Mai 2010, mis en ligne le 30 septembre 2010, consulté le 19 avril 2019. URL : http://journals.openedition.org/developpementdurable/8413; DOI : 10.4000/ developpementdurable.8413

Ce document a été généré automatiquement le 19 avril 2019 


\title{
Nouvelles formes de gouvernance dans le domaine de l'eau. Apports et limites de la coopération décentralisée dans les pays en développement
}

\author{
New Forms of Governance in the Water Sector. Contributions and Limitations of
} Decentralized Cooperation in Developing Countries

Raoudha Makkaoui et Jean Luc Dubois

1 Devenue ${ }^{1}$ un bien rare et inégalement répartie, l'eau dans les pays en développement (PED) se trouve être maintenant au cœur des préoccupations des décideurs politiques et de la communauté internationale. Le Forum mondial de l'eau, qui s'est tenu du 16 au 22 mars 2009 à Istanbul a insisté sur la gravité des problèmes liés à l'eau. Les enjeux humains, sanitaires et sociaux qui en dérivent, particulièrement pour les pays en développement (PED), sont en effet considérables. Or, la communauté internationale s'était engagée en 2000 lors du Sommet du Millénaire de New York à « (...) réduire de moitié, d'ici à 2015, la proportion des personnes qui n'ont pas accès à l'eau potable ou qui n'ont pas les moyens de s'en procurer " (ONU, 2000). Ces engagements, réaffirmés lors du Sommet de Johannesburg sur le développement durable en $2002^{2}$, ont relancé les actions de solidarité en matière d'accès à l'eau potable et à l'assainissement.

2 Selon le rapport mondial des Nations Unies sur la mise en valeur des ressources en eau (UNESCO, 2009), un habitant de la planète sur cinq n'a pas accès à l'eau potable et $40 \%$ de la population mondiale ne dispose pas d'un service d'assainissement de base (collecte et traitement des eaux usées). Compte tenu de l'augmentation prévue de la population, il faudrait, pour atteindre l'objectif de la déclaration du Millénaire, desservir 1,5 milliard de personnes supplémentaires, soit 100 millions de personnes de plus chaque année. Quant aux services d'assainissement, ce sont 2 milliards de personnes de plus, soit annuellement 
125 millions de personnes qui devraient pouvoir en bénéficier chaque année avant 2015. Bien qu'ambitieux, ces objectifs restent en deçà des besoins réels des populations vivant dans les PED. Or, le rapport du panel mondial sur le financement des infrastructures de l'eau montre que, pour atteindre ces objectifs, il faudrait envisager un montant d'investissement qui varierait entre 10 et 50 milliards de dollars pour tenir compte, à la fois, de la disponibilité et de la nature des ressources en eau, des technologies de desserte choisies et des milieux urbains ou ruraux à desservir.

3 La qualité de l'eau et son mode d'accès ont, de plus, un impact important sur la santé. En effet, une eau de mauvaise qualité peut provoquer de nombreuses maladies. Ainsi, selon le rapport des Nations Unies de 2009, plus de cinq millions de personnes meurent chaque année de maladies provoquées par une eau impropre à la consommation humaine et, parmi ces victimes, $90 \%$ sont des enfants de moins de cinq ans. Une situation alarmante, qui concerne surtout les populations des PED où l'accroissement démographique, combiné à une urbanisation insuffisamment maîtrisée et au développement de l'agriculture irriguée, a exacerbé le problème de l'eau.

4 C'est en 1978, lors de la Conférence de Mar del Plata, que fut initiée la décennie internationale de l'eau potable et de l'assainissement, permettant à de nombreuses rencontres internationales de se saisir de ces préoccupations afin de faire avancer le débat sur les problèmes liés à l'utilisation, à la disponibilité et à la qualité de l'eau potable. Son objectif était d'assurer à la population mondiale un accès à une eau potable de qualité, et en quantité suffisante, ainsi qu'à des installations sanitaires de base. Cependant, malgré des investissements de près de 100 milliards de dollars et le fait que, au cours de cette période, 1,3 milliards de personnes supplémentaires ont été desservies en eau potable et 750 millions en assainissement, les objectifs globaux n'ont pu être atteints. En 1990, 244 millions de citadins étaient toujours dépourvus d'accès à l'eau potable, ce qui représentait 30 millions de plus qu'en 1980 (Evans, 1992).

5 La déclaration de New Delhi (1990), clôturant cette décennie internationale, en soulignait l'échec et préconisait un changement d'orientation pour les politiques publiques correspondantes ${ }^{3}$. Dès le début des années 1990, des forums, conférences et rencontres internationales se sont multipliés pour élaborer une nouvelle vision de la gestion des ressources en eau qui soit partagée par tous les acteurs impliqués, afin de sensibiliser les décideurs économiques et obtenir des engagements concrets pour résoudre les problèmes de l'eau et de l'assainissement. Les discussions ont porté sur l'ouverture à de nouvelles sources de financement et sur l'implication correspondante de la communauté internationale et des sociétés civiles.

6 Dans ce contexte, la France a, par exemple, mis en place à partir de 1992, un cadre juridique et réglementaire permettant aux collectivités territoriales de promouvoir des relations de coopération directes avec les collectivités des pays en développement. Ces programmes sont répertoriés sous la dénomination de « coopération décentralisée ».

7 Les programmes de coopération décentralisée permettent de développer des synergies entre différents acteurs et de promouvoir des processus d'action sous une forme participative. La société civile devient ainsi partie prenante dans la conception et la mise en place de projets de coopération. Cette vision novatrice et complémentaire de la coopération internationale a le mérite de donner un nouvel élan aux politiques de développement. Elle permet, en effet, de dépasser les limites des dispositifs classiques de coopération bilatérale ou multilatérale entre pays du Nord et du Sud. 
On peut, dès lors, se demander si ces innovations institutionnelles fourniront une réponse appropriée au problème crucial du financement d'un accès universel à l'eau et à l'assainissement. D'autant plus qu'il faut maintenant non seulement assurer, de manière équitable, un droit d'accès à l'eau pour tous, mais aussi veiller à ce que cet accès permette d'accroître, de manière tout aussi équitable, les « capabilités » individuelles et collectives des bénéficiaires de l'eau. Par "capabilités", on entend, tout à la fois, les capacités d'action actuelles des bénéficiaires et leurs alternatives de choix dans le futur.

La problématique de cet article porte donc sur le rôle de la coopération décentralisée dans le domaine de l'eau, dans la mesure où celle-ci semble pouvoir apporter des réponses concrètes et constituer une nouvelle option pour la communauté internationale. Une option qui aurait pour finalité d'assurer de manière soutenable un accès universel à l'eau potable et à l'assainissement afin que, par ce biais, il soit possible d'atteindre les objectifs de bien-être souhaités par tous, en termes individuels comme collectifs.

On procédera donc en deux temps. Dans une première partie, seront examinés les différents modes de gestion de l'eau en tant que ressource rare (gestion publique, partenariat public-privé, approche participative) et la façon dont ces modes de gestion ont cherché à satisfaire des objectifs bien précis. Dans une deuxième partie, sur la base d'une initiative de coopération décentralisée concrète, on s'interrogera sur les apports et limites de cette nouvelle forme de partenariat participatif afin d'en évaluer la pérennité ainsi que la "soutenabilité sociale », montrant, de ce fait, sa proximité avec l'économie solidaire.

\section{1. Évolution des formes de gouvernance dans le domaine de l'eau au cours des années 1980-2000}

11 Différents modes de gestion ont été successivement mis en place au cours de ces dernières années pour tenter de gérer, de la manière la plus efficace et équitable possible, une ressource qui devenait relativement plus rare tout en faisant face à des financements de plus en plus réduits. Le choix initial du mode de gestion public permettait d'assurer un accès universel et équitable à l'eau potable, cette dernière étant considérée comme un bien public. L'appel au secteur privé, qui a suivi, s'est traduit par l'instauration de partenariats public-privé avec pour objectif d'accroître l'efficacité des financements devenus insuffisants, en cherchant à diminuer les coûts économiques pour une qualité de service égale. Enfin, le recours actuel aux différentes formes de gestion participative, au sein desquelles s'inscrit la coopération décentralisée, poursuit dans la même voie tout en visant à mieux répondre aux aspirations et besoins des populations. $\mathrm{Si}$, parmi ces trois modes de gestion de l'eau, les deux premiers - le mode de gouvernance publique et le partenariat public-privé - ont largement fait leurs preuves avec les avantages et les inconvénients que l'on connait, le troisième qui fait référence à une plus grande participation des acteurs sociaux dans la prise des décisions n'est actuellement qu'en pleine émergence. Il se construit sur la recherche de solutions pertinentes à définir en fonction des besoins et, en ce sens, est source d'innovation permanente. 


\subsection{La gouvernance publique des services de l'eau dans les PED : un certain constat d'échec}

12 Depuis ses débuts, et jusqu'à tout récemment, la gestion des ressources naturelles dans les PED et, particulièrement, celles des services de l'eau était le fait du secteur public qui, sous contrôle de l'État, s'appuyait sur des instruments économiques et réglementaires spécifiques comme, par exemple, la tarification au coût marginal. L'intervention publique était alors caractérisée par un fort niveau d'investissement et une priorité à la desserte en eau potable. La plupart des services d'eau potable ont suivi un schéma caractérisé par trois critères : premièrement, la propriété et l'exploitation publiques des infrastructures hydrauliques étaient assurées par des entreprises nationales en situation de monopole ; deuxièmement, la gestion était fortement centralisée, l'État assurant les fonctions de régulation de la gestion et de planification des investissements; et, troisièmement, les usagers avaient un rôle très réduit, la gestion publique ne prévoyant pas la participation des acteurs locaux ni celle des usagers (Makkaoui, 2009). Dans ce cadre, la tarification des services d'eau était du ressort des autorités publiques. Le tarif était déterminé en fonction de critères sociaux ou du développement de certaines activités économiques. La recherche d'universalité et d'équité socio-spatiale en termes d'accès aux services de l'eau s'est alors traduite par la mise en place de dispositifs d'accès libres et gratuits à des points d'eau collectifs, comme les bornes-fontaines publiques, accompagnés de structures tarifaires à effet redistributif telle que la tarification progressive sur la base de tranches sociales subventionnées. Ce mode de gestion a pu s'étendre jusqu'à la fin des années 1970, en raison de l'abondance des financements extérieurs dont bénéficiaient les pays en développement, le financement des infrastructures correspondantes étant essentiellement assuré par des dons ou des prêts orientés vers des projets spécifiques.

Cependant, au début des années 1980, avec la combinaison de l'explosion démographique, se traduisant par une urbanisation non maîtrisée dans les grandes villes, et du déficit des finances publiques, la gestion publique des services de l'eau et de l'assainissement de nombreux pays s'est trouvée confrontée à une sérieuse crise financière. Les investissements et la maintenance des infrastructures qui garantissaient la desserte en eau et en assainissement sur une bonne partie des quartiers défavorisés n'a plus été assurée. Cette situation, venait du fait que les conditions de l'offre de financement des pays industrialisés se sont modifiées à la suite du premier choc pétrolier. À la hausse des taux d'intérêt des prêts octroyés aux PED s'est alors rajoutée la baisse des termes de l'échange, le tout contribuant à l'accroissement de leur déficit public.

Le second choc pétrolier, en ralentissant les économies des pays développés, a encore réduit les débouchés commerciaux des PED et perturbé leurs équilibres macroéconomiques, entraînant des difficultés de paiement pour les pays les plus endettés. Il en est résulté un tarissement des sources de financement externes et une crise des finances publiques, alors que le renouvellement et l'extension des réseaux nécessitaient des investissements supplémentaires que les gouvernements des PED n'étaient plus en mesure d'assumer. Le manque de financement dans les infrastructures a entrainé de nombreux dysfonctionnements au niveau de la qualité et de la fiabilité des services qui se sont traduits par une pression insuffisante de l'eau, notamment dans les immeubles, des interruptions dans la délivrance des services et de longs délais de réparation en cas de panne. 
15 En terme de mesure, l'indicateur de référence pour apprécier la desserte du service d'eau à domicile était alors le taux de connexion des ménages au réseau public. Ainsi, dans les années 1990, les taux de connexion des grandes villes des PED étaient de $50 \%$ à Bombay, $35 \%$ à Madras, $13 \%$ à Port-au-Prince, etc. Ces taux demeuraient inférieurs à $50 \%$ dans la plupart des grandes villes d'Afrique de l'Ouest et d'Amérique latine (Mathys et Savina, 1994; Idelovitch et Ringskog, 1997). Ils étaient bien moindres dans les quartiers défavorisés des grandes villes et dans les centres urbains secondaires. Cet indicateur a été remplacé par le «taux d'accès acceptable à l'eau potable», qui englobe à la fois l'approvisionnement par branchement à domicile et les modes de desserte collective comme les bornes-fontaines. Selon ce taux, $80 \%$ des habitants de Buenos Aires et $70 \%$ de ceux de Tunis sont desservis en eau potable mais seulement $49 \%$ des habitants de Karachi et $29 \%$ des Luandais le sont (Jaglin, 2001). Plus généralement, l'accès acceptable à l'eau potable concernait, en 2000, $62 \%$ des Africains, $81 \%$ des asiatiques et $93 \%$ des LatinoAméricains (OMS, 2000).

16 Notons cependant que si cet indicateur retrace assez bien le niveau d'extension du service à domicile, il n'en reflète ni la qualité, ni le degré de fiabilité. Or, une mauvaise qualité du service, caractérisée, par exemple, par la fourniture discontinue de l'eau ou par des variations dans son débit, amène la population à élaborer des stratégies alternatives au réseau de distribution afin d'améliorer son accès à l'eau. Des stratégies qui incluent l'accroissement des capacités de stockage, l'usage de pompes de succion permettant d'extraire du réseau des volumes d'eau plus importants, ou même le retour aux puits en les équipant de pompes manuelles ou motorisées. Or, ceci a pour effet d'accentuer le phénomène de gaspillage, car les quantités d'eau ainsi prélevées sont souvent supérieures aux besoins réels et une partie de l'eau stockée est par la suite rejetée pour éviter qu'elle ne devienne stagnante.

\subsection{L'appel au secteur privé pour constituer des partenariats public- privé}

Les conséquences opérationnelles sur le secteur de l'eau potable de cette crise des finances publiques ont conduit à rechercher de nouveaux modes de gouvernance des ressources en eau. Plusieurs gouvernements ont opté pour une participation du secteur privé dans la gestion des services de l'eau potable et de l'assainissement. Les institutions financières internationales, particulièrement la Banque Mondiale, ont alors présenté cette réponse institutionnelle comme une alternative pertinente à la gestion publique. Cette nouvelle forme de régulation était supposée améliorer tout à la fois l'efficacité dans l'allocation des ressources et le financement des travaux de construction et de renouvellement des infrastructures relatives à l'eau et à l'assainissement (Rivera, 1996; Alcazar et al., 2002). Aussi, entre 1990 et 2000, plus de 200 programmes de partenariats public-privé ont été mis en œuvre dans près de 43 pays, contre seulement 8 entre 1980 et 1990 (Breuil, 2004).

18 On distingue généralement trois formes contractuelles de partenariat public-privé dans le domaine des services de l'eau. Il y a, tout d'abord, les contrats de service (la soustraitance et la gérance) qui consistent à transférer, sur une courte période (de 1 à 3 ans) et à un opérateur privé, un certain nombre de responsabilités liées à des activités spécifiques ou ponctuelles telles que les opérations d'entretien des infrastructures et le remplacement des compteurs d'eau. Ce type de contrat est en vigueur à Gdansk (Pologne), 
à Luanda (Angola) ainsi qu'à Mexico (Mexique). Il y a, ensuite, les contrats d'affermage : les ouvrages et les équipements nécessaires à l'exploitation du service sont financés et réalisés par l'État qui en délègue l'exploitation et l'entretien à un opérateur privé pour une période déterminée et pour un prix convenu. Ce type de partenariat est appliqué au Sénégal, en Jamaïque et en Guinée. Enfin, il y a les contrats de concession : l'opérateur privé est responsable non seulement de l'exploitation, de la maintenance et de la gestion, mais prend également à sa charge les investissements pour les extensions du service et des infrastructures, pour la durée du contrat. En d'autres termes, l'entreprise privée finance, réalise et exploite les équipements nécessaires (usines de potabilisation ou d'épuration, réseaux de distribution d'eau potable ou de collecte des eaux usées). À la fin du contrat de concession, l'opérateur est tenu de transférer ces équipements aux collectivités locales. La durée du contrat est alors fonction de la durée d'amortissement des ouvrages financés par le délégataire (de 20 à 30 ans). Il s'agit de la forme contractuelle de partenariat dominante dans le secteur de l'eau et de l'assainissement. Ainsi, $69 \% \mathrm{du}$ montant des investissements totaux réalisés par le secteur privé le sont dans le cadre d'un contrat de concession (Banque Mondiale, 2003). Au cours de la décennie 1990-2000, plusieurs pays d'Amérique latine ont fait appel à des opérateurs privés pour gérer leurs services de l'eau. C'est le cas de l'Argentine, la Bolivie, le Brésil, le Chili, la Colombie, l'Équateur, le Honduras, le Mexique, le Nicaragua, Panama, le Pérou, la République dominicaine, le Salvador et l'Uruguay. La plupart de ces opérateurs sont les filiales de l'une des trois grandes multinationales de l'eau : Suez, Vivendi et RWE-Thames Water.

19 L'engouement suscité par les partenariats public-privé au cours des années 1990 s'explique par la conjonction entre un contexte idéologique, marqué par les théories préconisant de reconsidérer le rôle de l'État, et la nécessité de trouver de nouvelles sources de financement.

La vague d'enthousiasme qui a accompagné la mise en place des premiers projets de partenariat public-privé dans les pays en développement a cependant été rapidement suivie par une déception tout aussi forte de la part des opérateurs privés et des autorités publiques, que de la part de la Banque Mondiale. Pourtant cette dernière avait fortement recommandé ce nouveau mode de gouvernance. Le partenariat public-privé dans le secteur de l'eau a été à l'origine de sévères critiques sur la difficulté de concilier la logique de l'action publique avec celle des interventions privées. En effet, l'action publique s'inscrit dans le cadre d'un contrat social qui vise à la réduction de la pauvreté et au développement durable en évitant de porter atteinte aux principes d'équité et d'universalité dans l'accès à l'eau, alors que la logique privée se situe, quant à elle, dans le cadre de l'économie de marché en visant surtout à la maximisation des profits.

Dans un article consacré au fonctionnement des contrats de concessions en Amérique latine, Estache et al. (2003) montrent que si des gains d'efficacité ont bien été partagés entre les gouvernements et les opérateurs, ils n'ont jamais bénéficié aux usagers. D'autres auteurs (Ménard et Shirley, 2002 ; Harris, 2003 ; Izaguirre, 2004) ont mis en évidence la faiblesse du cadre juridique et institutionnel dans un contexte macro-économique instable. Se rajoutent, la présence de structures tarifaires inadaptées et un problème plus général d'acceptabilité sociale. Les mises en application des partenariats public-privé ont, en effet, provoqué dans nombre de pays de vives contestations qui ont eu pour conséquence de les remettre en question (Makkaoui, 2009). Cela explique les nombreuses ruptures qu'ont connues les PED dans les contrats de concession de l'eau potable et de l'assainissement. Une large majorité de ces contrats a du être renégociée dans les quatre 
premières années. Selon Guasch et al. (2003), $76 \%$ des concessions d'eau en Amérique latine ont fait l'objet de renégociations en moyenne un an et demi après la signature du contrat. Ces renégociations sont notamment dues à des objectifs contractuels trop ambitieux et à une structure tarifaire inadéquate. On peut signaler, à cet égard, l'augmentation considérable du nombre d'arbitrages internationaux entre investisseurs privés et gouvernements des pays. Selon le Centre International pour le Règlement des Différends relatifs aux Investissements (CIRDI), plus de 26 cas de contentieux ont été enregistrés pour la seule année 2003, alors que seulement 21 cas d'arbitrages avaient eu lieu entre 1966 et 1986. En 2003, on dénombrait 140 contrats débouchant sur des litiges liés aux partenariats public-privé pour l'ensemble des pays en développement (CIRDI, 2003). Si bien que $40 \%$ des partenariats public-privé mis en place entre 1990 et 2003 ont été annulés ou sont en procédure d'arbitrage international pour le secteur de l'eau et de l'assainissement (Breuil, 2004).

Le fait que plusieurs expériences de partenariats public-privé en matière de services d'eau et d'assainissement aient échoué, comme ceux de Buenos Aires en Argentine ou de Cochabamba en Bolivie, fait ressortir certaines constantes. Tout d'abord, la responsabilisation du secteur privé au sein des partenariats s'avère trop souvent imparfaite car, lors des ruptures de contrat, c'est le secteur public qui demeure le fournisseur en dernier recours pour le service. Ensuite, les changements techniques (réhabilitation des réseaux) et institutionnels (redéfinition des règles d'appropriation, création de nouvelles institutions) induits par la mise en place des partenariats publicprivé ont pour effet de redéfinir les enjeux économiques, ce qui peut être à l'origine de conflits sociaux.

Ces échecs ont montré le besoin d'une démarche plus participative qui puisse associer l'ensemble des composantes de la société civile à la gestion des ressources, dans le cadre de la redéfinition des relations entre l'État et le secteur privé. Mais ceci implique d'élaborer de nouveaux dispositifs de gestion de l'eau afin de pallier aux problèmes d'acceptabilité sociale posés par les partenariats public-privé, tout en faisant face à la complexité croissante de l'approvisionnement en eau potable. Il en résulte une nouvelle génération de mesures de politique environnementale qui, prenant la forme de dispositifs hybrides, s'inscrit dans une démarche participative où interviennent, à côté des autorités publiques, des acteurs issus de la sphère marchande comme de la société civile.

\subsection{L'émergence de nouveaux modes de gestion de l'eau faisant appel à une démarche participative}

24 Le principe central de toute démarche participative est dual. D'une part, la participation des acteurs dans la formulation des politiques de l'environnement favorise l'appropriation des décisions par ces derniers. Cela permet d'aboutir à des choix socialement plus acceptables et d'atteindre effectivement les objectifs fixés. D'autre part, en facilitant l'implication des divers acteurs, les autorités améliorent la qualité de la base informationnelle, ce qui leur permet d'envisager de nouvelles modalités de compréhension du problème. L'élément commun de ces dispositifs réside dans le fait qu'ils valorisent l'échange d'informations, la négociation, le compromis et les engagements volontaires.

Parmi les arguments avancés par les défenseurs de la démarche participative comme mode de gestion durable des ressources, figure l'importance du capital social au sein des 
communautés (Wade, 1988 ; Ostrom, 1990 ; Baland et Platteau, 1996). En s'appuyant sur les relations de confiance mutuelles, d'échange et de réciprocité, le capital social contribue au renforcement des démarches participatives et permet de limiter les risques de comportements opportunistes. Les réseaux, les valeurs et les convictions communes, facilitent en effet la coopération au sein de groupes et, en permettant de réduire les contrôles entre différents membres, permet de limiter les coûts de transaction. La reconnaissance, et l'application d'un système de normes de jugement et de sanctions sociales, contraignent les individus à se comporter conformément aux « règles" de la communauté.

L'application de la démarche participative au domaine de l'eau a commencé au début des années 1990 et a conduit à une mutation dans la gestion des services de l'eau, ceux-ci passant d'une gouvernance fortement centralisée à une gouvernance communautaire. Cette transformation n'a cependant été ni improvisée, ni spontanée. Les institutions financières internationales, Banque Mondiale et FMI, ont joué un rôle primordial. Dans le domaine de l'irrigation, par exemple, la participation des communautés d'usagers est devenue une condition sine qua non pour l'accord de toute aide financière internationale.

Cependant, toute démarche participative n'est pas la panacée en matière de prise de décision dans le domaine des ressources environnementales. Elle présente certaines difficultés et des risques de détournement. Selon Van den Hove $(2001: 80)$ : «Ceux-ci ne sont pas des propriétés intrinsèques des approches participatives, mais correspondent à des situations d'inadaptation de l'approche choisie à son contexte ». La mise en application de procédures participatives ne garantit pas pour autant la réalisation des objectifs attendus et ne mène pas forcément aux solutions les meilleures. Dans certains cas, les approches participatives peuvent provoquer des effets inverses à ceux escomptés et même entraîner un blocage du processus décisionnel (Van den Hove, 2001).

Le désengagement de l'État de la gestion des services de l'eau, en particulier, revêt un caractère complexe car le transfert des responsabilités résultant d'une redéfinition des rôles respectifs de l'État, des opérateurs privés et des communautés locales, peut provoquer des tensions dans les relations entre ces trois pôles d'acteurs. En effet, d'une part, le fait de redéfinir les responsabilités des agents administratifs et de modifier leurs conditions socio-économiques par des procédures participatives, provoque un profond sentiment d'incertitude personnel et professionnel. D'autre part, l'efficacité de ces procédures dépend largement de la contribution, et même de l'adhésion, des différents services de l'État chargés de la gestion des ressources en eau (Corkery, 1995). D'autres difficultés sont inhérentes à la complexité des configurations politiques locales car les communautés d'usagers se situent souvent dans un environnement complexe et potentiellement conflictuel. Elles sont soumises à des jeux d'influences et d'intérêts complexes qui conditionnent les relations qu'entretiennent les populations locales entre elles et avec leur environnement. Lemarchand (2006) précise à ce sujet « Le village est un champ politique complexe, traversé d'influences et d'intérêts divers, où se côtoient le monde de l'invisible et du visible, les sorciers et les guérisseurs, les gens du pouvoir et les gens de la terre, les autochtones et les allogènes, les éleveurs et les cultivateurs ». Il existe ainsi une asymétrie notoire concernant le pouvoir d'influence des différents acteurs.

C'est dans cette mouvance d'idées, en matière de développement durable et de proximité résidentielle, que s'inscrivent les programmes de coopération décentralisée.

Notons que même si les moyens financiers d'action et les modalités d'intervention des acteurs concernés par cette "nouvelle initiative » ne se situent pas au même niveau que 
les deux premiers modes de gouvernance exposés dans cet article, nous considérons que les projets mis en oeuvre dans le cadre de ces programmes représentent bien une émanation et une illustration de la démarche participative dans la mesure où ils dépassent le spectre des actions d'aide humanitaire, du bénévolat ou de la coopération technique. Ces projets visent en effet à associer les acteurs concernés par la formulation de politiques publiques relatives à l'environnement, en considérant les différentes dimensions économiques, sociales et écologiques, afin de favoriser une certaine appropriation des décisions par ces derniers. Comme le précise Bocar Sissé et al. (1999) : « L'objectif de la coopération décentralisée est avant tout d'assurer un meilleur développement par une plus grande prise en compte des besoins et priorités exprimés par les populations; elle vise ainsi à renforcer le rôle et la place de la société civile locale dans le processus de développement. Elle associe et fait collaborer à différents niveaux d'intervention les acteurs territoriaux tant du Nord que du Sud. Elle suscite la participation active et déterminante des bénéficiaires aux prises de décisions et aux différentes étapes des actions qui les concernent ".

On peut donc se demander si cette démarche qui fait de plus en plus appel à la société civile permet un accès plus durable à l'eau potable et à l'assainissement dans les pays en développement. Ceci implique d'examiner les apports et les limites de ces nouvelles approches sur la base de quelques critères qu'il convient de dégager à partir d'exemples concrets. Et ainsi, plus précisément, de faire ressortir les conditions d'une durabilité sociale qui se trouve être en permanence confrontée aux contraintes économiques, notamment financières, et écologiques, car propres à la disponibilité de l'eau.

\section{La coopération décentralisée comme illustration de la démarche participative}

32 Il existe deux grandes acceptions du concept de coopération décentralisée : l'une est française, l'autre européenne.

33 Au sens français, la coopération décentralisée est définie par la loi du 6 février 1992 de la façon suivante : «Il y a coopération décentralisée lorsqu'une (ou plusieurs) collectivité(s) locale(s) française(s) développe(nt) des relations avec une (ou plusieurs) collectivité(s) locale(s) étrangère (s): il peut s'agir aussi bien de l'établissement de relations d'amitié ou de jumelage avec des collectivités territoriales étrangères, d'actions de promotion à l'étranger, d'aide au développement de collectivités dans certains pays, d'assistance technique, d'action humanitaire, de gestion commune de biens de services, mais aussi de coopération transfrontalière et de coopération interrégionale » (CNCD, 2000). Dans les faits, les programmes de coopération décentralisée ne concernent que les collectivités territoriales mais sont particulièrement développés dans le domaine de l'eau et de l'assainissement, car les collectivités territoriales françaises peuvent bénéficier de deux types de dispositifs. En premier lieu, la loi de 1992 qui a donné aux collectivités territoriales la possibilité de signer des accords de coopération avec des autorités locales étrangères. Elle a été complétée, le 27 janvier 2005, par la loi dite "Oudin-Santini» qui autorise les collectivités locales, syndicats intercommunaux et agences de l'eau à consacrer jusqu'à $1 \%$ du budget annexe de leurs services d'eau et d'assainissement au financement d'actions de coopération internationale dédiées au secteur de l'eau.

34 Pour la Commission européenne, les actions de coopération décentralisée sont plutôt vues comme un moyen de s'engager vers "une autre façon de faire de la coopération » qui se doit 
d'impliquer les acteurs de la société civile, prise dans toute sa diversité (Commission européenne, 1999). L'acception européenne de la coopération décentralisée s'avère donc légèrement différente de la vision française dans le sens où elle n'implique pas forcément la présence d'une (ou de plusieurs) collectivité(s) locale(s). L'Union européenne reconnaît, en effet, l'ensemble des acteurs non étatiques tels que les ONG, les coopératives, les institutions d'enseignement et de recherche, les entreprises privées, etc., et les encourage à intervenir dans de tels programmes.

Notons toutefois que malgré ces différences de définition originelles, les deux approches convergent fortement lors de leur mise en application. Dans les deux cas, il s'agit bien de sortir du système des coopérations centralisées menées avec les États. Un bon exemple est fourni par le Conseil général du département de Seine-Saint-Denis qui réalise plusieurs actions de coopération décentralisée avec la ville de Figuig au Maroc, le district du Setùbal au Portugal, la ville de Matola au Mozambique et les villes autonomes palestiniennes de Djenin, Qalqilya et Tulkarem situées au nord de la Cisjordanie. Toutes ces réalisations impliquent des acteurs forts différents, car à côté des collectivités locales françaises et étrangères directement engagées dans les opérations, ont été sollicités des associations de jumelage, de migrants, de solidarité internationale, mais aussi des établissements de santé, des organismes de financement publics nationaux et multilatéraux, des organismes de recherche, des pôles universitaires et des centres de formation. Il en résulte qu'il ne s'avère pas forcément nécessaire d'effectuer une distinction, en termes opérationnels, entre les visions française et européenne. Et ce d'autant plus que la collectivité locale, en étant l'initiateur du programme de coopération décentralisée, devient le maître d'ouvrage et définit le mode de fonctionnement et d'organisation institutionnelle; ce dernier pouvant alors prendre de multiples formes comme des jumelages ou jumelages-coopération, des programmes ou projets de développement, des échanges techniques, etc. Les actions internationales sont alors pilotées par la ville elle-même qui les intègre dans son service de relations internationales, sous la responsabilité d'un élu. Il arrive aussi qu'une partie, sinon la totalité de l'action de coopération soit déléguée, par convention précisant les objectifs et le budget, à un organisme tiers qui peut être une association, une ONG ou encore un comité de jumelage. En fait, on peut remarquer qu'au-delà de ces distinctions conceptuelles, les deux formes de coopération sont, dans la pratique, souvent astucieusement combinées, qu'importe la forme d'organisation locale retenue. Une collectivité dotée, sous le contrôle d'un élu, d'un service de relations internationales et coopération décentralisée, pourra donc confier une mission de coopération à un acteur local particulier comme Cités-Unies France, par exemple, rejoignant ainsi la vision européenne de la coopération décentralisée.

Nous nous proposons d'examiner, à titre d'exemple, l'expérience de coopération décentralisée actuellement menée dans les secteurs de l'eau et de l'assainissement par le Conseil général du département de Seine-Saint-Denis en partenariat avec la ville de Figuig, une commune du Maroc.

\subsection{Gérer l'eau à Figuig : une expérience de gestion décentralisée}

37 La ville de Figuig est située au bord d'une oasis du Sud-est marocain et compte près de 12000 habitants selon le recensement de 2004. Longtemps isolée en raison de sa position géographique excentrée, de la fermeture de la frontière algérienne et de son faible niveau 
de développement, Figuig jouit aujourd'hui d'une vitalité associative étonnante. Elle compte plus de 40 associations comme la caisse des écoles, l'association des résidents des 7 ksour pour le développement, les associations de parents d'élèves, d'handicapés, de diabétiques, etc. Cette mobilisation de la population ne fait que perpétuer l'ancienne tradition berbère de travail collectif qui est caractéristique de l'identité berbère. La population locale possède un savoir-faire traditionnel concernant l'action collective qui s'exprime à travers les D'jmaas, ces assemblées représentant les habitants d'un ksar. L'austérité économique qui résulte d'un fort chômage des jeunes, de conditions climatiques extrêmes (une sécheresse sévère et un processus de désertification qui s'accélère) et de l'isolement géographique ont renforcé l'esprit de solidarité entre les résidents de cette région. Les résidents conservent un sentiment de fierté et d'attachement intense à cet héritage culturel, renforcé par le fait qu'un certain nombre de leurs parents ont dû émigrer au cours des décennies passées.

Un protocole de coopération a été signé le 8 juin 2000 entre la ville de Figuig et le département de Seine-Saint-Denis. Il portait sur le développement des services publics relatifs à l'environnement, la santé et l'emploi ${ }^{4}$. Un certain nombre de priorités ont été définies entre les partenaires, parmi lesquelles figurent les actions du volet environnement relatives à «la gestion de l'eau et la lutte contre la désertification ». Ce dernier prévoyait différentes interventions relatives au secteur de l'eau: une amélioration de l'accès à l'eau potable en utilisant le réseau existant, la mise en place d'un réseau d'assainissement, et la lutte contre la désertification. La construction de ce réseau d'assainissement vise à limiter la contamination des zones aquifères et la pollution du sol causée par les puits perdus et les puisards où les eaux usées de la ville sont déversées sans traitement.

L'objectif de cette initiative est de promouvoir «un mode de coopération propice à un

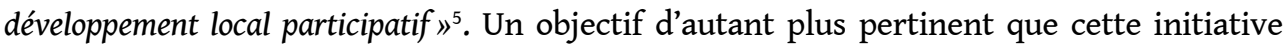
rencontre un écho très favorable au sein de l'importante communauté originaire du Maroc, et notamment de Figuig, qui réside en Île-de-France dans les départements de la Seine-Saint-Denis et du Val-de-Marne. En septembre 2001, plusieurs associations animées par des originaires de Figuig qui militaient pour le développement de cette région se sont regroupées afin de créer la Fédération des associations de Figuig en France (Fafef) ${ }^{6}$. En avril 2003, une convention quadripartite a été signée, associant le conseil général de Seine-Saint-Denis et la ville de Figuig au Syndicat Intercommunal pour l'Assainissement de l'Agglomération Parisienne (Siaap) et à la Fafef), afin de démarrer la réalisation du réseau de collecte des eaux usées et la mise en place de la station d'épuration par lagunage.

Sur le terrain, la Fafef s'est positionnée comme un partenaire de soutien, d'accompagnement et de mise en relation, révélant les acteurs économiques et sociaux capables de mener les opérations et de conduire les compromis sociaux inévitables pour ce type de coopération. Elle a ainsi directement contribué à la conception du réseau d'assainissement en montant un groupe de réflexion sur la santé qui rassemblait les professionnels du Conseil général de Seine-Saint-Denis, des militants associatifs et des professionnels de la santé originaires de Figuig.

41 De telles actions facilitent la mise en relation des différents acteurs locaux, autorités publiques, agents des collectivités, membres des D'Jmâas, etc., avec leurs partenaires du Nord, chercheurs, experts et techniciens, agents des services départementaux de SeineSaint-Denis, etc. Ainsi, se sont engagés dans ce programme au côté de la Fafef, des 
bureaux d'études techniques (Sol-paysage et Hydrasol), des chercheurs de l'INRA, des organismes publics comme le Siaap et la Direction de l'Eau et de l'Assainissement (Dea). L'échange des savoirs respectifs contribue au renforcement des capacités techniques et organisationnelles des opérateurs de la ville de Figuig. Cette démarche est complétée par des actions ciblées de formation professionnelle destinées aux élus et au personnel de la ville de Figuig. Deux personnes ont ainsi suivi une formation sur la gestion en régie d'un service de l'eau, organisée par la municipalité de Tremblay-en-France, seule ville du département de Seine-Saint-Denis disposant d'un service d'eau en régie (Le Cam, 2006).

Concernant la réalisation du réseau d'assainissement, une première phase de construction a été achevée en 2006 avec six kilomètres de collecteurs installés permettant le raccord au réseau de 2500 personnes. La deuxième phase est maintenant en cours, la ville de Figuig se faisant appuyer dans ce domaine par le conseil général de la Seine-SaintDenis et la Fafef. En ce qui concerne la lutte contre la désertification, des actions visant à économiser l'eau ont été engagées en partenariat avec le bureau d'études Hydrasol. Elles ont pour but de rationaliser la consommation d'eau en utilisant des « tensiomètres » et en formant le personnel aux techniques correspondantes. Les autres projets sont en phase de conception, ou en début de réalisation, comme la construction de la station de lagunage, la réutilisation des eaux traitées pour l'agriculture et la mise en régie du service public de l'assainissement.

\subsection{Comment analyser la soutenabilité sociale d'une telle expérience?}

La question se pose alors est de savoir évaluer la soutenabilité sociale d'une telle expérience. Autrement dit, il s'agit de voir dans quelle mesure cette nouvelle forme de gestion participative s'avère durable en termes sociaux.

Les expériences passées ont montré que la gestion publique qui visait un accès universel à l'eau et à l'assainissement était finalement trop coûteuse en termes d'infrastructures et de maintenance face aux capacités financières des États. D'un autre côté, la gestion en partenariat public-privé, qui devait améliorer l'efficacité dans l'allocation des ressources et ainsi permettre le renouvellement des infrastructures, s'est heurtée à un problème d'acceptabilité sociale, en raison des inégalités qu'elle engendrait. En ouvrant maintenant sur une base participative, la gestion de l'eau et de l'assainissement à la coopération décentralisée, on tente de mieux répondre aux aspirations et besoins de la population tout en satisfaisant aux contraintes économiques, notamment financières, et aux obligations écologiques. Des contraintes financières que l'on se propose de surmonter par une gestion plus efficace et mieux concertée. Des obligations écologiques qui concernent la préservation d'une eau rare et non polluée et qui ont besoin de la présence de municipalités, voire de l'État, pour garantir un droit universel et équitable d'accès à l'eau.

Cette approche des problèmes de l'eau et de l'assainissement s'inscrit dans la vision du « développement durable» telle que définie par le rapport Brundtland comme un mode de développement qui répond aux besoins des populations présentes sans compromettre la capacité des générations futures à répondre à leurs propres besoins (CMED 1989). Dans ce cadre, il semble naturel de se demander si l'ensemble des réalisations déjà effectuées par la ville de Figuig, de même que celles qui encore en cours de réalisation, respectent bien les critères qui fondent la durabilité du développement. 

la durabilité, notamment en termes sociaux. Le premier concerne la capacité des populations à satisfaire à leurs besoins actuels comme futurs. Ce qui veut dire que même si les besoins futurs ne sont pas toujours clairement définis et doivent être mis en relation avec les aspirations des populations, il faut que soit assurée l'accessibilité de tous à l'ensemble des biens et services (ce qui inclut l'eau et l'assainissement). Le second critère soulève la question de la répartition des capacités entre différentes générations. Il aborde ainsi la question de l'équité dans la répartition des ressources disponibles au sein d'une même génération et entre générations. Ces deux conditions peuvent être considérées comme essentielles pour assurer la soutenabilité sociale de toute action de développement (Ballet et al. 2005).

la cause de frustrations extrêmes qui peuvent conduire à des révoltes et violences diverses, s'exprimant sous la forme de conflits armés, de partitions territoriales, etc. (Dubois 2006, Boussou 2008, Razakatiana 2008). distribution de ces capacités, de revenir aux aspirations des personnes pour définir les besoins, et d'examiner les droits d'accès à certains biens et services, conduit à se rapprocher de la vision théorique d'Amartya Sen (1990) afin d'élaborer, sur la base de son approche par les capabilités, les instruments que nécessitent l'évaluation de cette soutenabilité.

La notion de capabilité exprime le fait « d'être capable de ». Elle va au-delà de celle de capacité, ou de compétence, car elle retrace, non seulement ce qu'un acteur peut effectivement faire - c'est-à-dire sa capacité actuelle - mais aussi ce qu'il pourrait réaliser s'il le souhaite dès lors que l'opportunité lui en est donnée - autrement dit sa capacité potentielle. La notion de capabilité débouche ainsi sur la possibilité de choix entre différentes alternatives et retrace ainsi l'éventail des possibilités qu'un acteur est, ou serait, capable de faire ou d'être. Parmi ces alternatives, certaines sont effectivement réalisées et donc observables, alors que d'autres restent au niveau du choix potentiel et ne peuvent être estimées que par induction statistique. La capabilité retrace ainsi le niveau de «liberté de choix» d'un acteur parmi les différentes alternatives de réalisations qui s'offrent à lui (Bonvin et Farvaque, 2008). Un développement qui se veut durable viserait alors à améliorer les capabilités de la génération actuelle sans compromettre le renforcement des capabilités des générations futures (Sen, 2000).

notion de capabilité s'inscrit dans le raisonnement économique au travers d'une équation reliant les ressources aux capabilités. Chaque acteur utilise la dotation en ressources (biens, actifs, droits, etc.) dont il dispose pour la transformer en une série de fonctionnements, effectifs comme potentiels. Cela se fait au travers de contraintes et d'opportunités sociales comme économiques, en tenant compte des caractéristiques personnelles (sexe, âge, handicaps, qualités, etc.). L'ensemble des divers fonctionnements que cet acteur peut effectivement ou potentiellement réaliser exprime sa capabilité (par exemple, aller chercher de l'eau, de devenir médecin ou médiateur, participer à la vie collective ou animer une association, etc.). Une analyse économique conduite en ces termes demande de recueillir des informations sur les caractéristiques des personnes, leurs dotations, les contraintes et opportunités rencontrées, et la façon dont elles vont convertir leurs dotations en fonctionnements. 
51 Dans ce cadre, le développement a pour objectif d'accroître les capabilités des acteurs ou, autrement dit, l'éventail de leurs libertés de choix. Il faut veiller, cependant, à ce que cet accroissement se fasse de manière équitable pour assurer une égalité entre les capabilités des acteurs (Sen, 1979). Sous cette condition, le renforcement des capabilités peut contribuer à la mise en œuvre d'un développement qui soit durable en termes sociaux.

Comment alors relier ce cadre théorique avec la démarche participative qu'implique la coopération décentralisée? Un raisonnement équivalent peut être fait sur une base collective en considérant des groupes d'individus, sous forme d'associations ou même d'institutions. Cependant le processus qui permet la constitution d'une capabilité collective à partir de capabilités individuelles est assez complexe à analyser et ne peut se concevoir comme une simple agrégation. En effet, le niveau de capabilité collective dépend de la qualité des relations qui existe entre les acteurs. Des relations harmonieuses peuvent accroître la capacité d'action collective au-delà de la somme des capabilités individuelles, alors que l'apparition de conflits entre acteurs peut, à l'inverse réduire, à néant tout effet collectif. Il faut donc introduire des capabilités particulières, les capabilités sociales, qui retracent la qualité de l'interaction sociale caractérisant chaque personne dans sa relation à une autre. La capabilité collective résulte alors de la combinaison des capabilités individuelles et sociales (Dubois et al., 2008), sachant que des institutions de toutes sortes, y compris l'État, peuvent interférer dans le processus de constitution d'une capabilité collective et dans sa transformation en action collective.

Dans ce cadre théorique, on raisonne sur une "économie d'acteurs capables » au lieu de se cantonner, comme la vision économique classique, à la seule allocation des ressources et la gestion de biens et de services (Giraud et Dubois, 2008). On relie ainsi les analyses sur le niveau et les conditions de vie des économistes aux analyses sur les comportements et les manières de vivre des sociologues. Ainsi, l'approche par les capabilités offre un cadre unifié au travers duquel on peut traiter, d'un même coup, les questions relevant de la pauvreté, perçue comme privation de capabilité, des inégalités, en considérant la distribution des capabilités, de la vulnérabilité et de la résilience, en déterminant les manques spécifiques de capabilités. Même l'analyse de l'exclusion sociale peut s'y rattacher dès lors qu'elle résulte d'un défaut d'accessibilité généralisé et d'un déficit de capabilité d'accès aux biens et services clés.

Cette unification du cadre d'analyse est fort utile pour analyser la durabilité sociale des projets qui sont réalisés dans le cadre de la coopération décentralisée. En effet, il devient possible d'aborder d'un seul tenant les conséquences sociales qui peuvent résulter des interactions pas toujours prévisibles entre la réduction de la pauvreté, l'évolution des inégalités ou de la vulnérabilité, l'apparition de formes d'exclusion, etc. Certaines interventions qui permettent de réduire la pauvreté dans des domaines particuliers, comme c'est le cas pour un meilleur accès à l'eau potable et à l'assainissement, peuvent engendrer de la vulnérabilité ou des inégalités dans d'autres domaines en raison de situations d'éloignement ou de densité de population. D'où la nécessité d'analyser correctement la situation, d'examiner les conditions de soutenabilité correspondantes et de rechercher les principes éthiques de précaution ou de prudence sociale qui permettent d'anticiper de tels risques (Dubois et Mahieu, 2002). Ainsi, dans le cas de Figuig, on ne se préoccupera pas uniquement des aspects d'allocation et de gestion des ressources en eau, mais on examinera aussi les aspirations des acteurs, les formes d'accès à l'eau qui sont envisageables, l'accroissement des capabilités qui pourrait en résulter et leur répartition au sein de la population, les effets sur les comportements des différents acteurs, etc. 

entre les différentes dimensions, écologique, économique, et sociale. Nous avons certes privilégié, dans cet article, les conditions de durabilité sociale, mais il conviendrait aussi de tenir compte de l'impact des conditions de durabilité écologique et économique sur la durabilité sociale. Ainsi, la dimension écologique demande d'aborder les questions relatives à la qualité des eaux souterraines. En effet, la construction d'un réseau d'assainissement, d'une station d'épuration, le traitement des eaux usées et leur réutilisation en agriculture permettent de lutter contre la pollution. En terme de santé publique, cela évite la prolifération de maladies liées à l'eau contaminée et permet le développement d'activité de loisirs sur l'oasis. Et concernant l'écosystème, cela évite l'infiltration dans la nappe phréatique, la contamination des aquifères et des captages d'irrigation. tenir compte des besoins de financement annuels pour la réalisation des projets, des charges d'entretien et de maintenance qui en résultent de façon à éviter un endettement excessif tant pour les générations actuelles que futures. La recherche d'une répartition équitable dans la disponibilité, l'accessibilité et l'utilisation des infrastructures, est aussi un élément essentiel de durabilité économique. considérations d'ordre culturel, en tenant compte des traditions, religions et identités, et d'ordre politique pour la faisabilité de tels projets, en considérant les rapports de force et la mobilisation citoyenne. On peut, cependant, penser que de telles dimensions, au demeurant fort pertinentes, sont déjà prises en compte par le choix du mode de gouvernance retenu par la coopération décentralisée. En effet, c'est à la gouvernance participative qu'il revient de reconnaitre, en faisant appel au débat public, la contribution spécifique que peuvent apporter les dynamiques traditionnelles à la conception et la réalisation des projets de développement.

Tous ces éléments contribuent à l'analyse de la soutenabilité sociale. Ils demandent néanmoins que des instruments d'observation et d'analyse spécifiques soient mis en place, sous formes d'enquêtes ou d'observatoires, pour établir des indicateurs, simples ou composites, qui soient susceptible d'illustrer les principes de précaution ou de prudence sociale. Sur cette base, il devient alors possible de sonder le tissu social en parallèle à l'avancement des projets afin de suivre les perceptions qu'en a la population et la façon dont évoluent les comportements.

\subsection{Apports et limites de l'expérience de Figuig}

approche de l'évaluation de projets en termes de soutenabilité sociale, appliquée au cas du partenariat entre la ville de Figuig et le département de Seine-Saint-Denis, met en valeur les acquis possibles de la coopération décentralisée dans la gestion des services de l'eau et de l'assainissement. Elle soulève toutefois un certain nombre de questions qui amènent à s'interroger sur la pertinence d'interventions de coopération décentralisée équivalentes en d'autres endroits.

60 Le premier point fort de ce partenariat est qu'il s'inscrit dans une logique de subsidiarité qui permet de relier deux territoires du Nord et du Sud autour d'un problème commun à résoudre. La force de la coopération décentralisée est bien de favoriser la rencontre d'acteurs se situant à des niveaux de décisions équivalents, mais parallèles, pour décider 
de mesure appropriées, sans pour autant avoir systématiquement besoin de remonter au niveau de l'État, de la région ou de la province. Le fait de vouloir résoudre des problèmes équivalents dans des contextes différents encourage les échanges de savoirs et les transferts de technologie. Cela permet de porter un regard croisé sur les problèmes techniques, ce qui peut-être source de synergie et de créativité accrue.

Le deuxième point fort concerne l'émergence d'une vision de responsabilité partagée, ou "coresponsabilité », face aux nécessités du développement et à ses conséquences économiques, écologiques et sociales. Le partenariat entre les deux régions fournit des éléments au débat public et facilite l'émergence de politiques concertées au sein desquelles les sociétés civiles partenaires se trouvent, de fait, engagées. Leurs visions spécifiques, qui peuvent être tantôt complémentaires, tantôt opposées, contribuent à l'élaboration de méthodes d'approche et de processus de décision originaux.

Enfin, un troisième point concerne la question plus générale de l'émergence d'un nouveau «style de développement» qui exprimerait "une autre façon de faire de la coopération", selon les vœux européens. On peut alors se demander si la coopération décentralisée ne permet aux valeurs, qui sont habituellement portées par l'économie solidaire, de pénétrer la sphère de l'économie publique.

L'économie solidaire répond aux urgences du monde, qu'elles soient d'ordre humanitaire, écologique, ou de justice sociale. Elle fonctionne au sein d'un univers très hétérogène car mondialisé, où l'on est rarement " entre égaux solidaires pour agir » comme c'est le cas pour l'économie sociale, mais où il faut plutôt «agir ensemble de manière solidaire et comme des égaux, tout en étant fortement différents ». On intègre ainsi la question de la différence dans un cadre égalitaire. Dans ce contexte, le principe de réciprocité qui fonde l'économie solidaire devient un principe de partenariat qui s'appuie sur des critères éthiques particuliers : la responsabilité vis-à-vis des plus vulnérables, la recherche de l'équité et la reconnaissance de la différence. L'économie solidaire, en recherchant le bien commun des différents partenaires, privilégie l'intérêt général sur l'intérêt collectif de chaque partenaire, qui n'est alors considéré que comme groupe d'égaux solidaires. Et dans ce contexte, les valeurs de l'économie solidaire complètent les valeurs classiques de l'économie sociale qui sont la liberté, l'égalité, et la solidarité. Ainsi la liberté se double de la responsabilité avant l'action, la lutte contre les inégalités fait appel à l'équité, et la solidarité intègre la reconnaissance de l'autre (Dubois, 2009). Or, ce qui est intéressant, c'est de constater que la coopération décentralisée contribue, à travers ses réalisations, à la pénétration de ces trois valeurs de responsabilité, équité et reconnaissance de la dignité de l'autre, dans les politiques publiques de développement. Une dynamique qui pourrait être plus profondément analysée dans le cadre des interventions effectuées sur une base participative à Figuig.

64 Face à ces quelques points forts, on ne peut, cependant nier l'existence d'un certain nombre de difficultés qui risquent de porter atteinte à plus long terme, à la durabilité des interventions qui ont été réalisées ou sont en cours de réalisation. Ces difficultés étant, de plus, en interaction, il convient de les aborder de front pour pouvoir les résoudre.

Il y a, en premier lieu, des difficultés d'ordre technique, qui sont liées au manque de qualification professionnelle du personnel des associations participant au programme. Les associations marocaines sont souvent créées autour d'un objectif précis qui réduit leur capacité d'intervention. Comme elles privilégient généralement la dimension socioculturelle et investissent peu dans le domaine du développement économique, les 
actions de développement en partenariat international sont peu nombreuses, ce qui est le cas pour Figuig.

De plus, l'insuffisance d'informations permettant d'éclairer les décisions et le manque de communication entre les différents acteurs, rend difficile la prise de décision collective. Un rapport de la mission communication du département Seine-Saint-Denis à Figuig (2003) énonce : « l'information existante est vague, partielle et non officielle. La principale source d'information est le oui-dire (...)", ce qui a un impact sur la perception de la population visà-vis des projets qui sont mis en œuvre.

La question de l'adhésion de la population et de sa mobilisation face à la réalisation des différents projets est, en effet, la deuxième grande difficulté à laquelle se heurte le partenariat entre les deux collectivités. On peut examiner cette question de deux manières différentes: d'une part, en considérant les problèmes de gouvernance locale que cela pose, et, d'autre part, en examinant les contraintes financières qui en résultent.

Certaines associations sont accaparées par des querelles internes qui ralentissent ou même bloquent l'avancement des projets. Des querelles qui ne peuvent être facilement tranchées en raison du manque d'informations objectives et reconnues de tous. Se rajoute le manque de conviction de certains fonctionnaires qui demeurent sceptiques quant à l'efficacité de la coopération décentralisée. Comme le souligne Chambrin (2005): «ces initiatives se heurtent parfois à des a priori, voire des critiques de certains fonctionnaires qui considèrent ces actions comme inutiles, coûteuses et leurs déplacements comme période de villégiature ».

69 Plus graves sont les contraintes financières qui viennent du fait que les projets de coopération décentralisée s'appuient fortement sur la dynamique du réseau associatif. Or, les associations dépendent de leur capacité à mobiliser les fonds nécessaires à leur propre fonctionnement et à l'encadrement des projets dont elles portent la responsabilité. Il leur faut donc sans cesse rechercher de nouvelles sources de financements. Mais celles-ci sont porteuses de priorités qui obligent les associations à réorienter leurs activités en fonction de la stratégie des bailleurs de fonds. Et ces priorités ne sont pas toujours en résonnance avec les réalités locales et les besoins effectifs de la population.

70 Certes, on peut tenter de surmonter l'ensemble de ces difficultés en renforçant les capacités individuelles et collectives par des mesures appropriées, mais cela demeure une gageure. En effet, malgré la mise en place de dispositifs de formation en parallèle à l'avancement des projets, les résultats à moyen terme ne sont pas toujours à la hauteur des attentes. Ainsi, l'éventail des compétences requises pour réaliser un programme aussi important que celui de Figuig, s'étend du diagnostic institutionnel et organisationnel, à la collecte de l'information, la maitrise d'ouvrage, la gestion de services publics, la planification en concertation avec les habitants, etc. Force est alors de constater que l'ensemble de ces domaines couvre une étendue bien plus large que ce que proposent les actions de formation qui restent souvent ponctuelles et ciblées.

71 Autrement dit, parallèlement aux actions de formation, qui demeurent indispensables pour accroître les capacités individuelles et collectives, il faut mettre en place un suivi régulier des conditions de durabilité afin d'éviter les risques d'échec de telles expériences de partenariat. Ceci demande l'instauration de systèmes spécifiques de suivi, ou de veille, qui soient capables d'appréhender l'évolution de la situation sociale et de déceler l'émergence de phénomènes pouvant atteindre la durabilité sociale des projets en cours de réalisation. 


\section{Conclusion} partenariat, et que l'on considère les critères permettant de juger de la durabilité sociale des projets réalisés ou en cours de réalisation, on ne peut que s'interroger sur les risques sous-jacents. En effet, malgré des résultats positifs concernant la réduction de la pauvreté et l'amélioration du bien-être de la population, comme le prévoyaient les objectifs initiaux, demeurent les risques d'apparition de nouvelles inégalités sociales, ou de situation de vulnérabilité non initialement prévues, qui pourraient fragiliser le tissu social ou miner la cohésion sociale.

74 Certes, on ne peut éviter de tels risques. Ce qu'il faut, c'est observer régulièrement les impacts sociaux des projets réalisés, ou en cours de réalisation, au moyen de systèmes d'observation ou de veille adéquats qui fournissent des informations fiables et reconnues sur l'évolution de la situation. Ceci permet d'intervenir dès lors que certains des critères éthiques de précaution sociale se trouvent menacés. Dans un tel cadre, et à condition de déceler et de respecter les conditions de durabilité, les programmes de coopération décentralisée pourraient contribuer, en tant qu'étape instrumentale spécifique, à l'émergence d'un nouveau style de développement.

\section{BIBLIOGRAPHIE}

Alcazar L., Abdala M. et Shirley M., 2002, "The Buenos-Aires Water Concession", in Thirsting for efficiency, The Economics and Politics of Urban Water Reform, Elsevier Pub., pp. 65-102.

Baland J.-M. et Platteau J.-P., 1996, Halting degradation of natural resources : Is there a role for rural communities? Oxford, Clarendon Press. Disponible sur http://www.fao.org/docrep/x5316e/ x5316e00.htm.

Ballet J., Dubois J.-L. et Mahieu F.-R., 2005, L'autre développement : le développement socialement soutenable, Paris, L'Harmattan.

Bocar Sissé H., Idrissa Maiga S. et Bartholomeeussen S., 1999, « Lien entre la décentralisation et la coopération décentralisée au Mali, Document de réflexion $n^{\circ} 6$, Centre européen de gestion de politique de développement (ECDPM), Maastricht.

Développement durable et territoires, Vol. 1, n 1 | Mai 2010 
Bonvin J.-M. et Farvaque N., 2008, Amartya Sen : une politique de la liberté, Paris, Michalon.

Boussou V., 2008, «La crise ivoirienne : un exemple de non durabilité sociale », Qualitique : culture managériale du XIXème siècle. Business Ethics, $n^{\circ}$ 202, novembre, pp. 27-31.

Breuil L., 2004, Renouveler le partenariat public-privé pour la gestion des services d'eau dans les PEDComment conjuguer les dimensions contractuelles, institutionnelles et participatives de la gouvernance?, Thèse de gestion de l'ENGREF.

Chambrin M.H., 2005, Seine-Saint-Denis (France) - Figuig (Maroc) : avances et limites de la coopération, Mémoire de DESS, Université de Paris I.

CIRDI, 2003, Annual Report, Centre International pour le Règlement des Différends Relatifs aux Investissements, Washington, DC. Disponible sur http://www.worldbank.org/icsid/ pubs/1998ar/2003_ICSID_ar_en.pdf.

Cités-Unies France, L'organisation locale de la coopération décentralisée, Paris. Disponible sur http:// www.observ-ocd.org/temp/libreria-50_1.pdf.

Clément V., Le Clainche C. et Serra D., 2008, Théorie de la justice et de l'équité, Paris, Economica.

CMED (Commission mondiale sur l'environnement et le développement), 1989, Notre avenir à tous, Montréal, Éditions du Fleuve.

CNCD (Centre National de la Coopération Décentralisée), 2000, Guide de la coopération décentralisée, Paris : La Documentation française.

Commission Européenne, 1999, Note d'orientation sur la coopération décentralisée, Bruxelles. Disponible sur_www.europa.int.

Conseil général de la Seine-Saint-Denis, 2003, Rapport de la mission « communication » $d u$ département Seine-Saint-Denis à Figuig, Saint-Denis.

Corkery J., 1995, « Réforme du service public : obstacles et aides », Bulletin DPMN, vol. 3, n 1, pp. 9-11.

Dubois J-L., 2009, « La notion de style est-elle transposable au développement durable?» Transversalités $\mathrm{n}^{\circ} 109$ janvier-mars 2009, ICP, Paris, Desclées de Brouwer.

Dubois J.-L., Bakhshi P., Brouillet A.-S. et Duray-Soundrou C., 2008, Repenser l'action collective : une approche par les capabilités, Réseau IMPACT, Paris, L'Harmattan.

Dubois J.-L., 2006, « Approche par les capabilités et développement durable : La transmission intergénérationnelle des capabilités ", in V. Reboud (ed.) Amartya Sen : un économiste du développement?, Notes et documents $n^{\circ} 30$, AFD, pp. 201-213.

Dubois J.-L. et Mahieu F.-R., 2002, « La dimension sociale du développement durable : lutte contre la pauvreté ou durabilité sociale ? » in J.-Y. Martin (dir.), Développement durable ? Doctrines, pratiques, évaluations, Paris, IRD, pp. 73-94.

Evans P., 1992, Paying the Piper : an Overview of Community Financing of Water and Sanitation, Occasional Paper $n^{\circ} 18$, International Water and Sanitation Center, The Hague.

Estache A., Guasch J.-L. et Trujillo L., 2003, Price Caps, Efficiency Payoffs and Infrastructure Contract Renegotiation in Latin America, Washington D.C., World Bank, Policy Research Working Paper 3129. Giraud G. and Dubois J.-L., 2008, « Amartya Sen : l'économie d'acteurs capables », Projet n 306, Paris, pp. 4-11.

Guasch J.L., Laffont J.-J. et Straub S., 2003, Renegotiation of Concession Contracts in Latin America, Working Paper, University of Edinburgh. 
Harris C., 2003, Private Participation in Infrastructure in Developing Countries : Trends, Impact, and Policy Lessons, Washington, DC, World Bank Working Paper, $\mathrm{n}^{\circ} 5$.

Idelovitch E. et Ringskog K., 1997, Wastewater treatment in Latin America. Old and New Options. The World Bank, Washington, DC. Disponible sur http://www-wds.worldbank.org/servlet/ WDS_IBank_Servlet?pcont=details\&eid=000009265_3971229180845.

Izaguirre A. K., 2004, Private Infrastructure : Activity Down by $13 \%$ in 2003, Public Policy for the Private Sector, Washington D.C. The World Bank, note $\mathrm{n}^{\circ} 274$.

Jaglin S., 2001, «L'eau potable dans les villes en développement : les modèles marchands face à la pauvreté », Revue Tiers Monde, vol. 42 n 166, Paris, pp. 275-304.

Le Cam C., 2006, Co-développement et logiques participatives, les migrants dans la coopération décentralisée. L'exemple du partenariat entre le département de la Seine-Saint-Denis et la ville de Figuig (Maroc), Étude de cas - Master 2 professionnel « Administration des institutions et entreprises culturelles », Université de Paris.

Lemarchand R., 2006, «La Face Cachée de la Décentralisation : Réseaux, Clientèles et Capital Social ", Le bulletin de l'APAD, $n^{\circ} 16$, Décentralisation, pouvoirs sociaux et réseaux sociaux, mis en ligne le 6 octobre 2006. Disponible sur http://apad.revues.org/document522.html.

Makkaoui, R., 2009, Réflexion sur les formes d'action collective et la gouvernance des ressources en eau dans les pays en développement, thèse pour le doctorat en sciences économiques, Université de Versailles Saint-Quentin-en-Yvelines.

Mathys A. et Savina A., 1994, L'alimentation en eau en milieu urbain dans les quartiers défavorisés : une question de partage? GREA/Afrique de l'Ouest, Abidjan.

Ménard C. et Shirley M., 2002, « Cities Awash : a Synthesis of the Country Cases », in Shirley M., T hirsting for Efficiency - The Economics and Politics of Urban Water Reform, Pergamon Press, World Bank, pp. 1-41.

ONU (Organisation des Nations Unies), 2000, Déclaration du Millénaire des Nations Unies, 8 septembre 2000, New York. Disponible sur http://www.un.org/french/millenaire/ares552f.htm.

OMS (Organisation Mondiale de la Santé), 2000, Global Water Supply and Sanitation Assessment 2000, OMS, Genève. Disponible sur http://www.who.int/water_sanitation_health/monitoring/ jmp2000.pdf.

Ostrom E., 1990, Governing the Commons. The Evolution of Institutions for Collective Action, Cambridge, Cambridge University Press, xviii, 280 pages.

Rajaona K.D. et Dubois J.-L., 2008, «L'intérêt de l'approche par les capabilités pour le développement socialement durable », Qualitique : culture managériale du XIXème siècle. Business Ethics $\mathrm{n}^{\circ} 202$, novembre 2008, pp. 23-26.

Razakatiana S., 2008, «La politique de décentralisation malgache : moyen ou frein d'un développement socialement durable?", Qualitique : culture managériale du XIXème siècle. Business Ethics $n^{\circ} 202$, novembre 2008, pp. 32-35.

Rivera D., 1996, Private Sector Participation in the Water Supply and Wastewater Sector : Lessons from Six Developing Countries, The World Bank, Washington D.C.

Sen A.K., 2000, Social Justice and the Distribution of Income Handbook of Income Distribution, in A.B. Atkinson \& F. Bourguignon (ed.), Handbook of Income Distribution, edition 1, volume 1, chapter 1, pages 59-85 Elsevier. 
Sen A.K., 1999, Un nouveau modèle économique. Développement, justice, liberté. Trad. française Paris, Éditions Odile Jacob 2000.

Sen A.K., 1981, Poverty and Famines : An Essay on Entitlement and Deprivation, New York, Oxford University Press.

Sen A.K., 1979, Quelle égalité in Éthique et économie, et autres essais. Trad. française Paris, PUF, 1993.

UNESCO, 2009, Rapport mondial des Nations-Unies sur la mise en valeur des ressources en eau, «L'eau dans un monde qui change » UNESCO, Paris. Disponible sur http://www.unesco.org/ water/wwap/wwdr/wwdr3/index_fr.shtml.

Van den Hove S., 2001, « Approches participatives pour la gouvernance en matière de développement durable : une analyse en termes d'effets », in Froger, G. (Ed.), Gouvernance et développement durable, Bâle/Genève/Munich, Helbing \& Lichtenhahn, pp. 53-89.

Wade R., 1988, Village Republics : Economic Conditions for Collective Action in South India, vol. 11, Oakland, ICS Press.

\section{NOTES}

1. Les auteurs remercient les deux lecteurs anonymes pour leurs remarques et leurs suggestions sur une première version de ce texte.

2. Le Plan d'action adopté lors du sommet mondial pour le développement durable de Johannesburg affirme notamment: «nous convenons de réduire de moitié, d'ici à 2015, la proportion de personnes qui n'ont pas accès à l'eau potable ou qui n'ont pas les moyens de s'en procurer (comme énoncé dans les grandes lignes dans la Déclaration du Millénaire) et la proportion de personnes qui n'ont pas accès à des services d'assainissement de base ».

3. La déclaration de New Delhi concluait sur un bilan décevant de la décennie de l'eau et de l'assainissement et remettait en cause la manière d'utiliser les ressources financières : "Failure to achieve coverage targets in the 1980s has as much to do with the manner in which funding sources have been mobilized, allocated, and used as with the absolute level of resources available" (UNDP-World Bank and Sanitation Program 1990).

4. Précisons qu'outre les projets engagés avec le conseil général du département de Seine-SaintDenis, la ville de Figuig a également développé des relations de partenariat avec d'autres organisations de coopération et de solidarité internationale comme DANAFRICA Danemark, Croix-rouge France, CCFD France, USAID États-Unis, OXFAM Québec et la Chambre des Beaux Arts France.

5. Protocole de coopération décentralisée entre le Département de la Seine-Saint-Denis et la ville de Figuig, art. 3, pp. 2-3, annexe IX, p.XXV.

6. La fédération des associations de Figuig en France possède un site web : http://www.fafef.org. 


\section{RÉSUMÉS}

La problématique de cet article porte sur le rôle de la coopération décentralisée dans le domaine de l'eau, dans la mesure où celle-ci semble pouvoir apporter des réponses concrètes au problème de l'accès universel à l'eau et constituer une nouvelle option pour la communauté internationale. Dans une première partie, on présentera les problèmes rencontrés par les différents modes de gestion de l'eau dans les pays en développement et la façon dont ils ont successivement tenté de satisfaire à certains objectifs bien précis (accès universel à l'eau, réduction des coûts, prise en compte des besoins prioritaires). Dans une deuxième partie, sur la base d'une initiative de coopération décentralisée entre le Conseil régional de Seine-Saint-Denis et la ville marocaine de Figuig, on s'interrogera sur les apports et limites de cette nouvelle forme de partenariat participatif afin d'en évaluer la pérennité ainsi que la "soutenabilité sociale ", montrant, de ce fait, sa proximité avec l'économie solidaire.

Our paper builds on this issue of decentralized cooperation in the field of water management. Cooperation between decentralized entities in order to ensure the access to safe drinking water for all now constitutes in many places a preferred water governance option. Therefore, in the first part, we will provide an overview of the current difficulties encountered by the various forms of water management in developing countries, showing the solutions that they successively brought to specific issues (access for all, cost reduction, priority needs). In the second part, we will address the particular case of decentralized cooperation between the General Council of Seine-Saint-Denis in the suburbs of Paris, France, in partnership with the city of Figuig in Morocco. We review the insights and limits of such processes that refer to the people's capabilities, at both individual and collective levels, in order to ensure "social sustainability".

\section{INDEX}

Mots-clés : eau, gouvernance, coopération décentralisée, approche par les capabilités, soutenabilité sociale

Keywords : water, governance, decentralized cooperation, capability approach, social sustainability

\section{AUTEURS}

\section{RAOUDHA MAKKAOUI}

Raoudha Makkaoui est docteur en sciences économiques et chercheur associé au LADYSS (Laboratoire dynamiques sociales et recomposition des espaces). Ses travaux portent sur l'économie politique du développement et sur l'action publique environnementale mise en œuvre dans un domaine particulier, celui de la gestion de l'eau. Elle s'intéresse en particulier à 
l'étude des enjeux de pouvoir, des rapports de force et des formes de coordination dans la gouvernance des ressources en eau.r.mathieu@yahoo.fr

\section{JEAN LUC DUBOIS}

Jean-Luc Dubois est directeur de recherche à l'Institut de Recherche pour le Développement (IRD) et enseignant à l'Université de Versailles St Quentin en Yvelines. Il a été le président du Comité d'Orientation du Réseau IMPACT (appui aux politiques de lutte contre la pauvreté et les inégalités) entre 2006 et 2009 . Ses travaux portent les conditions de vie des ménages, l'approche par les capabilités, la pauvreté et la durabilité sociale, le questionnement éthique.

jldubois@aol.com 